\title{
MODELLING AND SIMULATION OF FOOD NETWORK
}

\author{
Tibor Kmet \\ Department of Computer Science \\ Faculty of Natural Sciences \\ Constantine the Philosopher University \\ Tr. A. Hlinku 1, 94974 Nitra \\ Slovak Republic \\ Email: tkmet@ukf.sk
}

\section{KEYWORDS}

Simple food web, partial functional differential equations, spatially constant equilibrium, stability analysis

\section{ABSTRACT}

The analysis of networks of ecological throphic transfers is a useful complement to simulation modelling in the quest for understanding whole-ecosystem dynamics. Throphic networks can be studied in quantitive and systematic fashion at several levels. The purpose of this paper is to investigate a system of parabolic equations with discrete time delays describing a simple throphic food network which consists of the $n+1$ type living organisms zooplankton, phytoplankton and microorganisms), the $n$ type dissolved organic and inorganic nutrients and detritus. When the delays are relatively small, our predictions are also identical to the predictions given by the corresponding PDE. The system of parabolic equations is discretized by the finite difference method which yields a coupled system of nonlinear algebraic equations. Stability analysis of equilibria and some numerical examples are given.
\end{abstract}

\section{INTRODUCTION}

In ecosystem modelling, food network design is a central task. Ecological networks are quantitative, graph-based descriptions of ecosystems, consisting of compartments (trophospecies and nutrient pools) that exchange fluxes of nutrient or energy. One approach that has been used to account for the lack of empirically derived data is the development of simple algorithms to construct hypothetical, but ecologically realistic networks. However, there have been two distinct approaches marked by the initial assumptions one makes. The first group, based on population/community ecology, focuses strictly on who eats whom, producing structures involving primary producers, grazers, and predators, but explicitly lacks decom- posers and detritus. As a result, these networks do not typically contain cycling. The second group, based on ecosystem ecology focuses on energy flowing the system and includes all functional groups including detritus (dead organic material) and decomposers (Fath at al., 2007). The interactions along the detritus feedback cycle are likely to occur at a different time scale than the conventional predation. This problem could be addressed by describing interactions with time-delayed differential equations (Halnes at al., 2007). The focus in most food web analyses has been on how they are arranged, affects different statistical and stability properties of model food webs. The dynamical theory of food webs has been based typically on local and global stability analysis, i.e. an existence and stability of equilibrum.

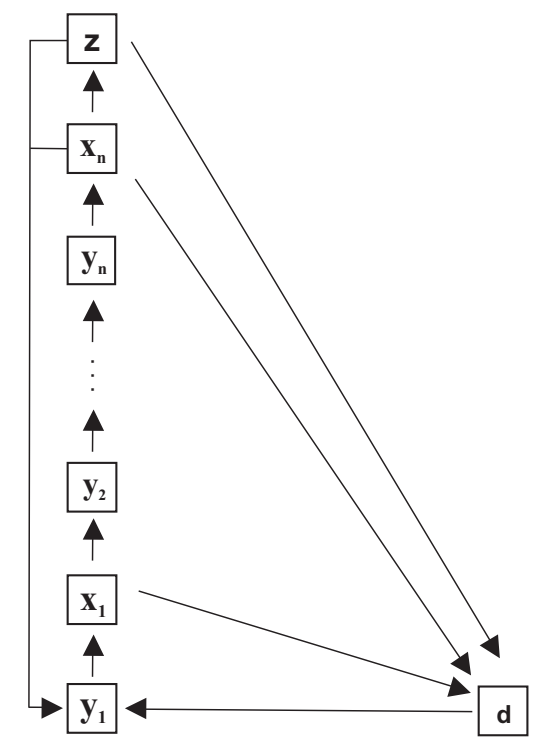

Figure 1. Diagram of the compartmental system modelled by (1): $x_{i}$, microorganisms; $x_{n}$, phytoplankton; $y_{i}$, nutrients; $d$, detritus; $z$, zooplankton. 
The model that we treat has $2 n+2$ components consisting of the $n+1$ type living organisms (zooplankton, phytoplankton and microorganisms), the $n$ type dissolved organic and inorganic nutrients and detritus as shown in Figure 1. The nutrient $y_{i}$, which is a metabolic product of the microorganisms $x_{i-1}$, is assumed to be the one primarily responsible for limiting the $x_{i}$ production for $i=2, \ldots, n-1$. The dissolved organic nutrient concentration $y_{1}$ is a result of the partial decomposition of the dead organisms. The phytoplankton $x_{n}$, which assimilates the metabolic product $y_{n}$, excretes the dissolved organic nutrient and limits zooplankton growth $(z)$. Zooplankton excretes dissolved organic matter, too. The $n+1$ living organisms levels, detritus and the $n$ nutrients are modelled in terms of their nitrogen content, $N$. We may assume that the material stream from the component $i$ to $j$ by the Lotka-Volterra hypothesis varies as the product of the interacting components. This quadratic assumption will cause the resulting differential equations to be nonlinear. The dynamics of a simple food network is described by the following functional reaction-diffusion equations:

$$
\begin{aligned}
& \frac{\partial d}{\partial t}=d_{d} \frac{\partial^{2} d}{\partial p^{2}}+\sum_{i=1}^{n} x_{i} M_{i}\left(y_{i}\right)+ \\
& z M_{z}\left(x_{n}\right)-K_{1} d\left(t-r_{k 1}, p\right) \\
& \frac{\partial x_{i}}{\partial t}=d_{x i} \frac{\partial^{2} x_{i}}{\partial p^{2}}+\quad x_{i}\left(t-r_{u i}, p\right) U_{i}\left(y_{i}\left(t-r_{u i}, p\right)\right)- \\
& x_{i}\left(t-r_{e i}, p\right) E_{i}\left(y_{i}\left(t-r_{e i}, p\right)\right)- \\
& x_{i}(t, p) M_{i}\left(y_{i}(t, p)\right) \\
& \frac{\partial x_{n}}{\partial t}=d_{x n} \frac{\partial^{2} x_{n}}{\partial p^{2}}+\quad x_{n}\left(t-r_{u n}, p\right) U_{n}\left(y_{n}\left(t-r_{u n}, p\right)-\right. \\
& x_{n}\left(t-r_{e n}, p\right) E_{n}\left(y_{n}\left(t-r_{e n}, p\right)\right)- \\
& x_{n}(t, p) M_{n}\left(y_{n}(t, p)\right)- \\
& z\left(t-r_{u z}, p\right) U_{z}\left(x_{n}\left(t-r_{u z}, p\right)\right) \\
& \frac{\partial z}{\partial t}=d_{z} \frac{\partial^{2} z}{\partial p^{2}}+ \\
& z\left(t-r_{u z}, p\right) U_{z}\left(x_{n}\left(t-r_{u z}, p\right)\right)- \\
& z\left(t-r_{e z}, p\right) E_{z}\left(x_{n}\left(t-r_{e z}, p\right)\right)- \\
& z(t, p) M_{z}\left(x_{n}(t, p)\right) \\
& \frac{\partial y_{1}}{\partial t}=d_{y 1} \frac{\partial^{2} y_{1}}{\partial p^{2}}+\quad z\left(t-r_{e z}, p\right) E_{z}\left(x_{n}\left(t-r_{e z}, p\right)\right)+ \\
& x_{n}\left(t-r_{e n}, p\right) E_{n}\left(y_{n}\left(t-r_{e n}, p\right)\right)+ \\
& K_{1} d\left(t-r_{k 1}, p\right)- \\
& x_{1}\left(t-r_{u 1}, p\right) U_{1}\left(y_{1}\left(t-r_{u 1}, p\right)\right) \\
& \frac{\partial y_{i}}{\partial t}=d_{y i} \frac{\partial^{2} y_{i}}{\partial p^{2}}+\quad x_{i-1}\left(t-r_{e i-1}, p\right) * \\
& E_{i-1}\left(y_{i-1}\left(t-r_{e i-1}, p\right)\right) \\
& -x_{i}\left(t-r_{u i}, p\right) U_{i}\left(y_{i}\left(t-r_{u i}, p\right)\right)
\end{aligned}
$$

with Neumann boundary condition

$$
\frac{\partial x_{i}}{\partial p}(t, 0)=\frac{\partial x_{i}}{\partial p}(t, 1)=0
$$

$$
\begin{aligned}
\frac{\partial y_{i}}{\partial p}(t, 0) & =\frac{\partial y_{i}}{\partial p}(t, 1)=0 \\
\frac{\partial d}{\partial p}(t, 0) & =\frac{\partial d}{\partial p}(t, 1)=0 \\
\frac{\partial z}{\partial p}(t, 0) & =\frac{\partial z}{\partial p}(t, 1)=0
\end{aligned}
$$

and initial conditions

$$
\begin{aligned}
x_{i}(t, p) & =\phi_{i}(p) \geq 0 \\
y_{i}(t, p) & =\phi_{n+i}(p) \geq 0 \\
d(t, p) & =\phi_{2 n+1}(p) \geq 0 \\
z(t, p) & =\phi_{2 n+2}(p) \geq 0,0 \leq p \leq 1, t \in\langle-r, 0\rangle
\end{aligned}
$$

where $x_{i}(t, p), y_{i}(t, p), i=1, \ldots, n, d(t, p)$ and $z(t, p)$ are the concentration of the recycling matter in microorganisms, the available nutrients, detritus and zooplankton, respectively. The constants $r_{* *}$ stand for the discrete time delays in uptake and excretion of nutrient and decomposition of detritus, $r=\max \left\{r_{* *}\right\}$ and $0<p<1$.

From the biological viewpoint the functions $F_{i}, U_{i}$, $E_{i}$ and $M_{i}$ describe the growth, uptake, excretion and mortality rate of the living organisms, respectively and there exists $\bar{y}_{i}>0$ such that $F_{i}\left(\bar{y}_{i}\right)=0$ and $F_{i}\left(y_{i}\right)\left(y_{i}-\bar{y}_{i}\right)>0$ for $i=1, \ldots, n$ and similarly there exists $\bar{x}_{n}>0$ such that $F_{z}\left(\bar{x}_{n}\right)=0$ and $F_{z}\left(x_{n}\right)\left(x_{n}-\bar{x}_{n}\right)>0$.

For detail explanation of function see (Kmet, 1996) and (Kmet, 2003).

In the previous work (Kmet, 1996) and (Kmet, 2003) we give a mathematical analysis of simple food web describing by ordinary differential equations and partial differential equations, respectively.

The global and local asymptotic behavior of the similar models of $n$ species of microorganisms competing exploitatively for a single growth-limiting nutrient was studied in (Busenberg at al., 1990), (Candaten at al., 2003), (Ellermeyer, 1994), (Nisbet at al., 1983) and (Wolkowicz, 1997).

Note 1 By Whu (1996) p. 37 Theorem 1.1 there exists unique mild solution of (1)-(3). Through the remainder of this paper, all solutions are mild solutions.

Note 2 Let us denote $X=\left(x_{1}, \ldots, x_{2 n+2}\right)=$ : $\left(x_{1}, \ldots, x_{n}, y_{1}, \ldots, y_{n}, d, z\right)$. For each continuous $\Phi=$ $\left(\phi_{1}, \ldots, \phi_{2 n+2}\right):\langle-r, 0\rangle X\langle 0,1\rangle \rightarrow R_{+}^{2 n+2}$, there is a unique function $X(\Phi,)=.\left(x_{1}(\phi,),. \ldots, x_{2 n+2}(\phi,).\right)$ called the solution of (1)-(3) through $\Phi$. The nonnegative cone of $C_{+}^{2 n+2}$ defined by 
$C_{+}^{2 n+2}=\left\{\begin{array}{c}\Phi \in C^{2 n+2} \mid \phi_{i}(t, p) \geq 0, \quad t \in\langle-r, 0\rangle \\ \text { and } p \in\langle 0,1\rangle \text { and } i=1, \ldots, 2 n+2\end{array}\right\}$

\section{PRELIMINARY RESULTS}

The following basic proposition holds

Proposition 1 The solution $X(., \Phi)=X(t, p)=$ $\left(x_{1}(t, p), \ldots, x_{2 n+2}(t, p)\right)$ of $(1)-(3)$ exists for all $\Phi \in$ $C_{+}^{2 n+2}$, remains nonnegative and for all $t>0$

$$
\int_{0}^{1}\left(\sum_{i=1}^{2 n+2} x_{i}(t, p)\right) d p=c
$$

where $c=\int_{0}^{1}\left(\sum_{i=1}^{2 n+2} \phi_{i}(p, 0)\right) d p$.

Proof 1 Local existence is standard (Wu, 1996). System of equations (1) describing the dynamic of recycling matter can be written in the form:

$$
\frac{d}{d t} x_{i}=d_{x i} \frac{\partial^{2} x_{i}}{\partial p^{2}}+G_{i}(x) \text { for } i=1, \ldots, 2 n+2 .
$$

Let us denote

$R_{+}^{2 n+2}=\left\{\left(x_{1}, \ldots, x_{2 n+2}\right) \mid x_{i} \geq 0\right.$ for $\left.i=1, \ldots, 2 n+2\right\}$

By straightforward calculation we get that $G_{i}\left(x_{1}, \ldots, x_{i-1}, 0, x_{i+1}, \ldots, x_{2 n+2}\right) \geq 0$ for $i=1, \ldots, 2 n+2$ and $x_{j} \geq 0$ for $j=$ $1, \ldots, i-1, i+1, \ldots, 2 n+2$. We obtain that the region $R_{+}^{2 n+2}$ is positively invariant for our system. By adding up of right-hand side of (1) we get $\sum_{i=1}^{2 n+2} G_{i}(x)=0$. Integrate over the interval $[0,1]$ to obtain an equation for the derivative of total mass of recycling matter

$$
\begin{gathered}
\frac{d}{d t}\left(\int_{0}^{1}\left(\sum_{i=1}^{2 n+2} x_{i}(t, p)\right) d p\right)= \\
\int_{0}^{1} \frac{d}{d t}\left(\sum_{i=1}^{2 n+2} x_{i}(t, p)\right) d p=\int_{0}^{1} \sum_{i=1}^{2 n+2}\left(\frac{d}{d t} x_{i}(t, p)\right) d p= \\
\sum_{i=1}^{2 n+2} \int_{0}^{1} d_{x i} \frac{\partial^{2} x_{i}(t, p)}{\partial z^{2}} d p= \\
\sum_{i=1}^{2 n+2} d_{x i}\left(\frac{\partial x_{i}}{\partial z}(t, 1)-\frac{\partial x_{i}}{\partial z}(t, 0)\right)=0 . \\
\text { It follows easily that } \int_{0}^{1}\left(\sum_{i=1}^{2 n+2} x_{i}(t, p)\right) d p=c \\
\text { and } c=\int_{0}^{1}\left(\sum_{i=1}^{2 n+2} \phi_{i}(0, p)\right) d p .
\end{gathered}
$$

Note 3 The nonnegative orthant is positively invariant for the model presented, and the initial value problem is well-posed in the sense that unique solutions exist for all $t>-r$ where $r=\max \left\{r_{* *}\right\}$ and depend continuously on the initial data and parameters.

Let us denote

$S_{c}^{2 n+2}=\left\{X \in C_{+}^{2 n+2}+/ x_{i} \geq 0, \int_{0}^{1}\left(\sum_{i=1}^{2 n+2} x_{i}\right) d p=c\right\}$

and

$S_{c}=\left\{(0, y, 0,0) \in S_{c}^{2 n+2} / y_{i}\right.$ are spatially constant $\}$.

Note that $S_{c}$ is a trivial spatially constant equilibrium set.

Proposition $2 S_{c}^{2 n+2}$ is a positively invariant set of system (1).

Proof 2 It follows from Proposition 1.

In this section we study the system $(1-3)$. We show that for $z=0$ there exists a strictly positive equilibrium point lying on $S_{c}^{2 n+1}$.

In the case of $z=0$ and $r_{* *}=0$ the system (1) has the form :

$$
\begin{aligned}
\dot{x}_{i}= & d_{x i} \frac{\partial^{2} x_{i}}{\partial p^{2}}+x_{i} F_{i}\left(y_{i}\right) \\
\dot{y}_{1}= & d_{y 1} \frac{\partial^{2} y_{1}}{\partial p^{2}}+x_{n} E_{n}\left(y_{n}\right)+ \\
& K_{1} d-x_{1} U_{1}\left(y_{1}\right) \\
\dot{y}_{i}= & d_{y i} \frac{\partial^{2} y_{i}}{\partial p^{2}}+x_{i-1} E_{i-1}\left(y_{i-1}\right)-x_{i} U_{i}\left(y_{i}\right) \\
\dot{d}= & d_{d} \frac{\partial^{2} d}{\partial p^{2}}-K_{1} d+\sum_{i=1}^{n} x_{i} M_{i}\left(y_{i}\right)
\end{aligned}
$$

where $K_{i}>0, \quad F_{i}=U_{i}-E_{i}-M_{i}, F_{i}(0)<0$, $F_{i}^{\prime}\left(y_{i}\right)>0, E_{i} \geq \epsilon_{i} U_{i}, U_{i} \geq E_{i}$ and there exists $\bar{y}_{i}$ such that

$$
F_{i}\left(\bar{y}_{i}\right)=0 \text { and } F_{i}\left(y_{i}\right)\left(y_{i}-\bar{y}_{i}\right)>0 .
$$

Let us denote $c_{1}=\sum_{i=1}^{n} \bar{y}_{i}$.

Proposition 3 For $z=0$ and $c>c_{1}$ there exists $a$ strictly positive spatially constant fixed point of (1) $\alpha^{*}=$ $\left(x^{*}, y^{*}, d^{*}\right)$ in $S_{c}^{2 n+1}$. 
Proof 3 As follows from (5) $y^{*}$ must satisfy $y_{i}^{*}=\bar{y}_{i}$ for $i=1, \ldots, n$. The other components of the fixed point $\alpha^{*}$ in the interior of $S_{c}^{2 n+1}$ are the strictly positive solutions of the following $n+1$ linear equations.

$$
\begin{aligned}
-U_{1}\left(y_{1}^{*}\right) x_{1}+E_{n}\left(y_{n}^{*}\right) x_{n}+K_{1} d & =0 \\
E_{1}\left(y_{1}^{*}\right) x_{1}-U_{2}\left(y_{2}^{*}\right) x_{2} & =0 \\
E_{i-1}\left(y_{i-1}^{*}\right) x_{i-1}-U_{i}\left(y_{i}^{*}\right) x_{i} & =0 \\
E_{n-1}\left(y_{n-1}^{*}\right) x_{n-1}-U_{n}\left(y_{n}^{*}\right) x_{n} & =0 \\
M_{1}\left(y_{1}^{*}\right) x_{1}+\ldots+M_{n}\left(y_{n}^{*}\right) x_{n}-K_{1} d & =0 .
\end{aligned}
$$

Let us consider the matrix $P=J+\rho I$, where $\rho>-\max J_{i i}$ and $J$ is a matrix of linear equations (6). $P$ is an irreducibile nonnegative matrix and so the machinery of the Frobenius theory of nonnegative matrix applies. Since $l J=0$, also

$$
l P=\rho l,
$$

where l denotes the vector all of whose entries are 1. By the Perron-Frobenius theory (Gantmacher, 1959) there is a unique positive right eigenvector $s$ associated with the eigenvalue $\rho$, i.e. $P s=\rho s$.

We can normalize to get

$$
\begin{aligned}
x_{i}^{*} & =\left(c-c_{1}\right) s_{i} / \sum_{i=1}^{n+1} s_{i} \\
d^{*} & =\left(c-c_{1}\right) s_{n+1} / \sum_{i=1}^{n+1} s_{i} .
\end{aligned}
$$

There holds $(J+\rho I)\left(x^{*}, d^{*}\right)=\rho\left(x^{*}, d^{*}\right)$, so $J(x, d)=0$ and $x_{i}^{*}>0, d^{*}>0$ for $c>c_{1}$ and $i=1, \ldots, n$. It is evident that

$$
x_{1}^{*}+\ldots+x_{n}^{*}+d^{*}=c-c_{1} .
$$

This proves the result.

\section{STABILITY ANALYSIS}

In this section we investigate the of spatially constant equilibria for (1). The system (1) always has a trivial equilibrium set $S_{c}$. If $c>c_{1}$ there exists a unique equilibrium $\alpha^{*}$. The stability of $\bar{x} \in S_{c}$ and $\alpha^{*}$ is determined by the following eigenvalue problem.

As the Laplace operator has eigenvalues $-k^{2},(k=$ $0,1, \ldots)$ with corresponding eigenfunction $\cos (k \pi x), \lambda$ is a characteristic value of (1) if and only if for some $k=0,1, \ldots$ satisfies the characteristic equation

$$
H(\lambda) \equiv \lambda I-k^{2} D-J(\bar{x})=0 .
$$

where $D$ is a diagonal matrix with diffusion coefficients and $J(\bar{x})$ is described in APPENDIX I.

Let us denote

$S_{0}=\left\{\begin{array}{c}(0, y, 0,0) \in S_{c}^{2 n+2} / y_{i} \text { are spatially constant } \\ \text { and } y_{i}<\bar{y}_{i} \text { for } i=1, \ldots, n-1\end{array}\right\}$.

and suppose that $r_{u i}=r_{e i}=: r_{i}$ for $i=1, \ldots, n, 2 n+$ 2. Let us suppose that $F_{n}\left(y_{n}\right)<0$ for all $y_{n} \geq 0$. Applying Theorem 1 to the eigenvalue problem we obtain

Proposition 4 If $K_{1}<\min \left\{k^{2} \pi^{2} d_{x 2 n+1}, \frac{\pi}{2 r_{k 1}}\right\}$ then 0 is characteristic value of the linearization of (1) at $\bar{x} \in$ $S_{0}$ with multiplicity $n$ and all other characteristic values have negative real parts.

Proof 4 Let $\bar{x} \in S_{0}$. With the translation $y=x-\bar{x}$ we can write the system (1) as

$$
\dot{y}=D \frac{\partial^{2} y}{\partial p^{2}}+D G(\bar{x}) y+N(y)
$$

$N(y)$ involves only the higher terms in $y_{1}, \ldots y_{2 n+2}$. The perturbation system of (1) and its associated eigenvalue problem are, respectively, written as follows:

$$
\dot{y}=D \frac{\partial^{2} y}{\partial p^{2}}+D G(\bar{x}) y
$$

$$
\lambda v=\left(-k^{2} \pi^{2} D+J\right) v, k=0,1, \ldots
$$

The eigenvalues of $-k^{2} \pi^{2} D+J(\bar{x})$ at $\bar{x} \in S_{0}$ are as follows

$$
\begin{array}{r}
l \lambda_{i, k}=e^{-\lambda_{i, k} r_{i}}\left(u_{i}-e_{i}\right)-m_{i}-d_{x i} k^{2} \pi^{2} \\
\text { for } i=1, \ldots, n \\
\lambda_{i, k}=-d_{x i} k^{2} \pi^{2} \\
\text { for } i=n+1, \ldots, 2 n . \\
\lambda_{2 n+2, k}=e^{-\lambda_{2 n+2, k} r_{2 n+2}}\left(u_{2 n+2}-e_{2 n+2}\right)-m_{2 n+2}- \\
-d_{x 2 n+2} k^{2} \pi^{2}
\end{array}
$$

Upon simplification, the characteristic equation becomes

$$
H(\lambda)=H_{1}(\lambda) H_{2}(\lambda) H_{3}(\lambda),
$$

where $H_{1}(\lambda)=\prod_{i=1}^{n, 2 n+2}\left(\lambda_{i, k}-e^{-\lambda_{i, k} r_{i}}\left(u_{i}-e_{i}\right)+m_{i}+\right.$ $\left.d_{x i} k^{2} \pi^{2}\right)$, 
$H_{2}(\lambda)=\prod_{i=n+1}^{2 n}\left(\lambda_{i, k}+d_{x i} k^{2} \pi^{2}\right)$,

$H_{3}(\lambda)=\left(\lambda_{2 n+1, k}+K_{1} e^{-\lambda_{2 n+1, k} r_{k 1}}+d_{x 2 n+1} k^{2} \pi^{2}\right)$.

Denoted the three factor of $H(\lambda)$ by $H_{1}(\lambda), H_{2}(\lambda)$ and $H_{3}(\lambda)$, respectively. The location of the roots of the quasi-polynomials $H_{1}(\lambda)$ and $H_{3}(\lambda)$ is accomplished by using the Pontryagins theorem. (Hale at al., 1993) contains a brief introduction of the Pontryagin's results, together with a proof of Theorem 1, (see APPENDIX II Theorem 1).

To apply the Theorem 1 to the quasi-polynomial $H_{3}(\lambda)$, we first let $\omega=r_{k 1} \lambda$ so that $\omega$ and $\lambda$ have real parts of the same sign. Multiplying both side of $H_{3}(\lambda)$ by $r_{k 1} e^{\lambda_{2 n+1, k} r_{k 1}}$ yields

$$
\left(\omega+d_{x 2 n+1} k^{2} \pi^{2} r_{k 1}\right) e^{\omega}+r_{k 1} K_{1}=0
$$

All roots of the equation

$$
\left(\omega+d_{x 2 n+1} k^{2} \pi^{2} r_{k 1}\right) e^{\omega}+r_{k 1} K_{1}=0
$$

have negative real parts if and only if

$$
\begin{gathered}
d_{x 2 n+1} k^{2} \pi^{2} r_{k 1}>-1, \\
d_{x 2 n+1} k^{2} \pi^{2} r_{k 1}+r_{5} K_{1}>0
\end{gathered}
$$

and

$$
r_{k 1} K_{1}<\zeta \sin (\zeta)-d_{x 2 n+1} k^{2} \pi^{2} r_{k 1} \cos (\zeta)
$$

where $\zeta$ is the root of $\zeta=-d_{x 2 n+1} k^{2} \pi^{2} r_{k 1} \tan \zeta$, $0<\zeta<\pi$, if $d_{x 2 n+1} k^{2} \pi^{2} r \neq 0$ and $\zeta=\frac{\pi}{2}$ if $d_{x 2 n+1} k^{2} \pi^{2} r_{k 1}=0$.

If $K_{1}<\min \left\{k^{2} \pi^{2} d_{x 2 n+1}, \frac{\pi}{2 r_{k 1}}\right\}$ inequalities are satisfied and $H_{3}(\lambda)$ has roots with negative real parts.

If $\bar{x} \in S_{0}$ then $u_{i}-e_{i}-m_{i}<0$ and by the similar way it can be shown, that all the roots of the following transcendental equations given by $H_{1}(\lambda)$ have negative real parts for all $i=1, \ldots, n, 2 n+2$.

The equation $H_{1}(\lambda)=0$ is treated similarly by multiplying both side by $r_{i} e^{\lambda_{i, k} r_{i}}$ to obtain

$$
\lambda_{i, k}-e^{-\lambda_{i, k} r_{i}}\left(u_{i}-e_{i}\right)+m_{i}+d_{x i} k^{2} \pi^{2}=0,
$$

for $i=1, \ldots, n, 2 n+2$

$$
\left(\omega+\left(d_{x i} k^{2} \pi^{2}+m_{i}\right) r_{i}\right) e^{\omega}-r_{i}\left(u_{i}-e_{i}\right)=0 .
$$

We can apply Theorem 1.All roots of the equation

$$
\left(\omega+\left(d_{x i} k^{2} \pi^{2}+m_{i}\right) r_{i}\right) e^{\omega}-r_{i}\left(u_{i}-e_{i}\right)=0 .
$$

have negative real parts if and only if

$$
\left(d_{x i} k^{2} \pi^{2}+m_{i}\right) r_{i}>-1,
$$

$$
\left(d_{x i} k^{2} \pi^{2}+m_{i}\right) r_{i}-r_{i}\left(u_{i}-e_{i}\right)>0
$$

and

$-r_{i}\left(u_{i}-e_{i}\right)<\zeta \sin (\zeta)-\left(d_{x i} k^{2} \pi^{2}+m_{i}\right) r_{i} \cos (\zeta)$

where $\zeta$ is the root of $\zeta=-\left(d_{x i} k^{2} \pi^{2}+m_{i}\right) r_{i} \tan \zeta$, $0<\zeta<\pi$, if $\left(d_{x i} k^{2} \pi^{2}+m_{i}\right) r_{i} \neq 0$ and $\zeta=\frac{\pi}{2}$ if $\left(d_{x i} k^{2} \pi^{2}+m_{i}\right) r_{i}=0$. The condition of Theorem 1 are satisfied that means all roots of $H_{1}(\lambda)=0$ have negative real parts. This prove the result.

\section{NUMERICAL EXAMPLE}

If $k=0$ then $\zeta=\frac{\pi}{2}$ and

$$
r_{k 1} K_{1}<\frac{\pi}{2}
$$

i.e. for

$$
K_{1}>\frac{\pi}{2 r_{k 1}}
$$

all spatially constant $\bar{x} \in S_{0}$ are unstable.

Let us consider $H_{3}(\lambda)$ as a complex function of two complex variables

$$
\mathcal{F}\left(\omega, K_{1}\right)=\left(\omega+d_{x 2 n+1} k^{2} \pi^{2} r_{k 1}\right) e^{\omega}+r_{k 1} K_{1}
$$

If we let $\omega=\alpha+i \beta$ and setting $\alpha=0$. By Theorem 1 we get that there exists

$$
K_{1}^{*}=\frac{\pi}{2 r_{k 1}}
$$

for which $H_{3}(\lambda)$ has root with zero real part. $\omega_{0}\left(K_{1}^{*}\right)$ is a simple eigenvalue, therefore $\frac{\partial \mathcal{F}\left(\omega_{0}, K_{1}^{*}\right)}{\partial \omega} \neq 0$, by the implicit function theorem we get that

$$
\begin{gathered}
R_{e}\left(\frac{\partial \omega\left(K_{1}^{*}\right)}{\partial K_{1}}\right)=R_{e}\left(-\frac{\frac{\partial \mathcal{F}\left(\omega_{0}, K_{1}^{*}\right)}{\partial K_{1}}}{\frac{\partial \mathcal{F}\left(\omega_{0}, K_{1}^{*}\right)}{\partial \omega}}\right)= \\
\frac{r_{k 1} *\left(\pi / 2-\cos \left(\omega_{0}\right)\right)}{\left\|e^{i \beta}+i \beta e^{i \beta}\right\|^{2}}>0 .
\end{gathered}
$$

Real part of eigenvalue $\omega=\alpha+i \beta$ crosses the imaginary axis transversely at $K_{1}^{*}$. For $k=0 \quad 0$ is the eigenvalue with multiplicity $n$ which is a dimension of the family of equilibria set $S_{0}$. According to Proposition 4 the rest eigenvalues have been found to have real parts negative.

Our numerical calculations are based on Proposition 4 and iterative schemes for numerical solution of system of nonlinear parabolic equations with discrete time delays presented by (Pao, 1996), (Pao, 
1999). Finite difference solution was constructed. To simulate iterative scheme of finite difference solution a routine using software Matlab was created.

The vector function $F=\left(F_{1}, \ldots, F_{2 n+2}\right)$ given by right-hand side of (1) is mixed quasimonotone and the Jacobi iterative scheme, which is unconditionally stable with respect to the mesh sizes can be applied for numerical solution of (1).

Mathematical model of nitrogen transformation cycle, which consists of nine partial functional differential equations with discrete time delays was used, (Kmet, 2002) to numerical calculation.

Decomposition rate of detritus $K_{1}$ and discrete time delay rate $r_{k 1}$ play a role of bifurcating parameters.

For

$$
K_{1}>\frac{\pi}{2 r_{k 1}}
$$

all spatially constant $\bar{x} \in S_{0}$ are unstable. Numerical calculations show that for $K_{1}$ near to $K_{1}^{*}$ periodic solution occurs (Figure 2).

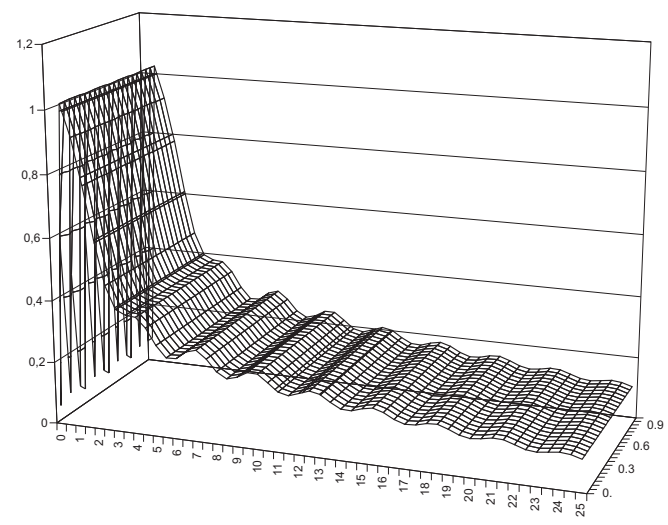

Figure 2. Numerical solution of (1) for $K_{1}=2.0$ and

$$
r_{k 1}=0.8\left(x_{1}(t, p)\right) \text {. }
$$

\section{If}

$$
K_{1} r_{k 1}<\frac{\pi}{2}
$$

all solutions converge to the set $S_{0}$ (Figure 3). If the time delay $r_{k 1}$ or the decomposition rate $k_{1}$ are relatively small $\left(K_{1} r_{k 1}<\frac{\pi}{2}\right)$, our predictions are identical to the predictions given by the corresponding PDE (Kmet, 2002).

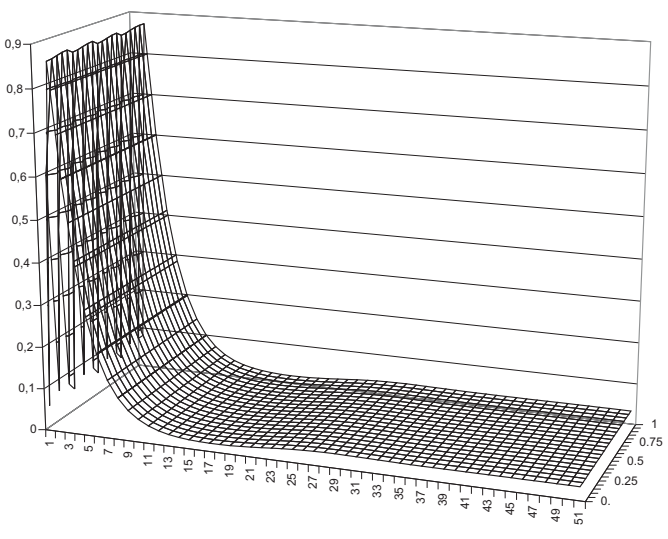

Figure 3. Numerical solution of (1) for $K_{1}=0.02$ and

$$
r_{k 1}=0.8\left(x_{1}(t, p)\right) \text {. }
$$

\section{CONCLUSION}

In this paper, we have first contructed a simple food network described by the time-delayed partial differential equations. Then we have verified the existence and stability of equilibria of food network. Finite difference solution was constructed. To simulate iterative scheme of finite difference solution a routine using software Matlab was created. Our simulation results demonstrate that periodic solution occurs.

\section{ACKNOWLEDGEMENTS}

We are grateful to anonymous reviewers for comments on the manuscript. The paper was worked out as a part of the solution of the scientific project number VEGA 1/4007/07 and KEGA 3/5277/07.

\section{APPENDIX I}

For $\bar{x} \in S_{c}$ the matrix $J(\bar{x})$ has the form:

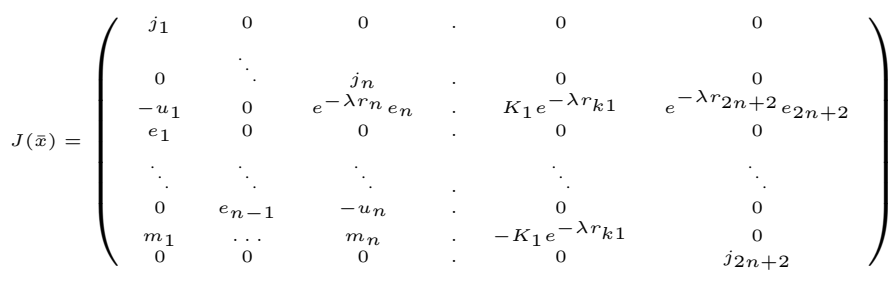

and $e_{i}=E_{i}(\bar{x}), m_{i}=M_{i}(\bar{x}), u_{i}=U_{i}(\bar{x}), j_{i}=$ $e^{-\lambda r_{i}}\left(u_{i}-e_{i}\right)-m_{i} i=1, \ldots, n$ and $2 n+2$. 


\section{APPENDIX II}

Theorem 1 All roots of the equation $(z+a) e^{z}+b=0$, where $a$ and $b$ are real, have negative real parts if and only if

$$
\begin{aligned}
a & >-1 \\
a+b & >0 \\
b & <\zeta \sin \zeta-a \cos \zeta
\end{aligned}
$$

where $\zeta$ is the root of $\zeta=-a \tan \zeta, 0<\zeta<\pi$, if $a \neq 0$ and $\zeta=\frac{\pi}{2}$ if $a=0$.

\section{REFERENCES}

Busenberg, S., Kumar, S. K., Austin, P., and Wake, G. (1990) The Dynamics of a Model of a Plankton-Nutrient Interaction. Bull. of Math. Biol., 52:677-696.

Candaten, M., and Rinaldi, S. (2003) Peak-to-paek dynamics in food chain models. Theoretical Population Biology, 63:257267.

Ellermeyer, S. F. (1994) Competition in the Chemostat:Global Asymptotic Behavior of a Model with Delayed Response in Growth. SIAM J. APPL. MATH., 54:456-465.

Fath, B. D., Scharler, U. M., Ulanowicz, R. E., and Hannon, B. (2007) Ecological network analysis: network construction. Ecological Modelling., 208:49 - 55.

Gantmacher, F. R. (1959) The theory of matrices. New York, Chelsea.

Hale, L. S., and Verduyn Lunel, S. M. (1993) Introduction to Functional Differential Equations. Springer Verlag, New York.

Halnes, G., Fath, B. D., and Liljenstrom, H. (2007) The modified niche model:Including detritus in simple structural food models. Ecological Modelling., 208:9 - 16.

Kmet, T. (1996) Material recycling in a closed aquatic ecosystem. I. Nitrogen transformation cycle and preferential utilization of ammonium to nitrate by phytoplankton as an optimal control problem. Bull. Math. Biol. , 58:957 - 982.

Kmet, T. (1996) Material recycling in a closed aquatic ecosystem. II. Bifurcation analysis of a simple food-chain model. Bull. Math. Biol. , 58:983 - 1000.

Kmet, T. (2002) Diffusive Mathematical Model of Nitrogen Transformation Cycle in Aquatic Environment. Folia Fac. SCi. Nat. Univ. Masarykiane Brunensis, Mathematica 11:105-114.
Kmet, T. (2003) Parabolic System Modelling a Simple Food Web in a Closed Aquatic Ecosystem. Proceeding $2^{\text {nd }}$ International Conference APLIMAT 149-158.

Nisbet, R. M., McKinstry, J., and Gurney, W. S. (1983) A "Strategic" Model of Material Cycling in a Closed Ecosystem. Mathematical Biosciences, 64:99 - 113.

Pao, C. V. (1996) Dynamic of Nonlinear Parabolic Systems with Time Delays. J. Math. Anal. Applic. , 198:751-779.

Pao, C. V. (1999) Numerical Methods for System of Nonlinear Parabolic Equations with Time Delays. J. Math. Anal. Applic. , 240:249-279.

Ulanowicz, R. E. (1972) Mass and Energy Flow in Closed Ecosystems. J. Theor. Biol., 34:239 - 253.

Wolkowicz, G. S. K., and Xia, H. Global Asymptotic Behavior of a Chemostat Model with Discrete Delays. SIAM J. APPL. MATH. , 57:1019-1043.

Wolkowicz, G. S. K., Xia, H., and Ruan, S. (1997) Competetion in the Chemostat:A Distributed Delay model and its Global Asymptotic Behavior. SIAM J. APPL. MATH. , 57:12811310.

Wu, J. (1996) Theory and Applications of Partial Functional Differential Equations. Spinger, Applied Math. Sciences, 119.

\section{AUTHOR BIOGRAPHIES}

TIBOR KMET was born in 1956. His email is tkmet@ukf.sk and his personal webpage is at http: //www.ki.fpv.ukf.sk. 